\title{
Oscillations in the magnetoconductance autocorrelation function for ballistic microstructures
}

\author{
Jian-Zhi Tang, Ludger Wirtz, and Joachim Burgdörfer* \\ Department of Physics, University of Tennessee, Knoxville, Tennessee 37996-1200 \\ and Oak Ridge National Laboratory, Oak Ridge, Tennessee 37831-6377 \\ Charles Marcus \\ Department of Physics, Stanford University, Stanford, California 94305
}

(Received 16 July 1997)

\begin{abstract}
We present a comparison between experiment and theory for the magnetoconductance autocorrelation function $C(\Delta B)$ for transport through a stadium-shaped ballistic microstructure. The correlation function displays damped oscillations which can be traced to the quantum interference between bundles of short trajectories. We present two different semiclassical calculations applicable for large and small mode numbers of the quantum wire, respectively. Good agreement is found with experimental data taken at relatively low mode numbers. [S0163-1829(98)03316-5]
\end{abstract}

Advances in semiconductor fabrication have made it possible to produce semiconductor devices of submicron size (see, e.g., Ref. 1). The size of such mesoscopic devices can be made small compared to the phase-coherence length $l_{\phi}$ due to inelastic electron-electron scattering at low temperatures $(\leqslant 0.1 \mathrm{~K})$ and smaller than the elastic electron mean free path. Transport through these devices is therefore ballistic and determined by the shape of the microstructure. The conductance through these open quantum dots is strongly influenced by quantum interference effects.

Recently, magnetoconductance measurements were performed for open quantum dots with simple geometric shapes such as a circle and a stadium. ${ }^{2-4}$ A stadium-shaped microstructure is of particular interest since the underlying classical dynamics of the ballistic electron motion is chaotic as known from investigations of the closed "Bunimovich" stadium. 5 The magnetoconductance displays strongly irregular but reproducible fluctuations which can be conveniently characterized by the magnetoconductance autocorrelation function $C(\Delta B)$.

Both experimental data as well as quantum calculations indicate that $C(\Delta B)$ displays oscillations with a negative overshoot and a partial revival of correlation as a function of magnetic field change $\Delta B$. These oscillations indicate that magnetoconductance fluctuations in ballistic quantum transport are far from being random but exhibit long-range correlations. Oscillations in $C(\Delta B)$ have been also observed in quantum calculations for a stadium with collinear leads at the straight sides. ${ }^{7}$ The understanding of structures in the autocorrelation function $C(\Delta B)$ is of importance since they are expected to be more robust and less sensitive to small imperfections of the device compared to the conductance fluctuations itself. Our goal in the following is to describe these oscillations semiclassically and to explain their classical origin.

Semiclassical theory provides a link between the classical dynamics of the electron motion in the cavity and quantum transport. It is well-suited to explore nonuniversal properties of the conductance fluctuations which originate from geometry-specific scattering processes. Several semiclassical approximations to quantum transport have been proposed..$^{7-14}$ We present in the following, to our knowledge, the first semiclassical description of oscillatory structures in $C(\Delta B)$. Oscillations result from interference between pairs of short trajectories connecting the entrance and the exit quantum wires. We employ a full evaluation of the sum over bundles of paths which goes beyond the customary primitive semiclassical (PSC) approximation by avoiding the stationary-phase approximation for the integration over the leads. This semiclassical (SC) approach is valid for lowmode numbers. In addition we implement a modified version of the PSC approximation, valid in the limit of large mode numbers, in which the classical trajectory Monte Carlo (CTMC) method is used for mapping between quantum numbers (or mode numbers) and the classical phase space. The latter approach provides an intuitive connection between the magnetoconductance oscillations and peaks in the classical area pair distribution.

An electron entering the stadium cavity from one quantum wire (entrance lead) will bounce, in general, several times off the walls of the cavity before exiting through the other quantum wire (exit lead) or being reflected back into the entrance lead. A typical trajectory in the presence of a perpendicular homogeneous magnetic field is shown in the inset of Fig. 3. Classical transport through open quantum dots is organized in terms of bundles of trajectories. All trajectories within a bundle are topologically equivalent, i.e., they can be generated by a continuous deformation of a single path having the same number of collisions with the boundary, an identical Maslov index $\mu_{\alpha}$ and smoothly varying action $F_{\alpha} \cdot{ }^{13}$ The semiclassical transmission amplitude between the incoming mode $m$ and outgoing mode $n$ can be written as a sum over bundles $\alpha$,

$$
\begin{aligned}
t_{m n}= & -\sqrt{v_{x, m} v_{x, n}} \frac{1}{(2 \pi i)^{1 / 2}} \sum_{\alpha} \exp \left[i \left(\bar{F}_{\alpha}\left(k_{F}, B\right)\right.\right. \\
& \left.\left.-\frac{\pi}{2} \mu_{\alpha}\right)\right] H_{\alpha}\left(k_{F}, m, n\right),
\end{aligned}
$$




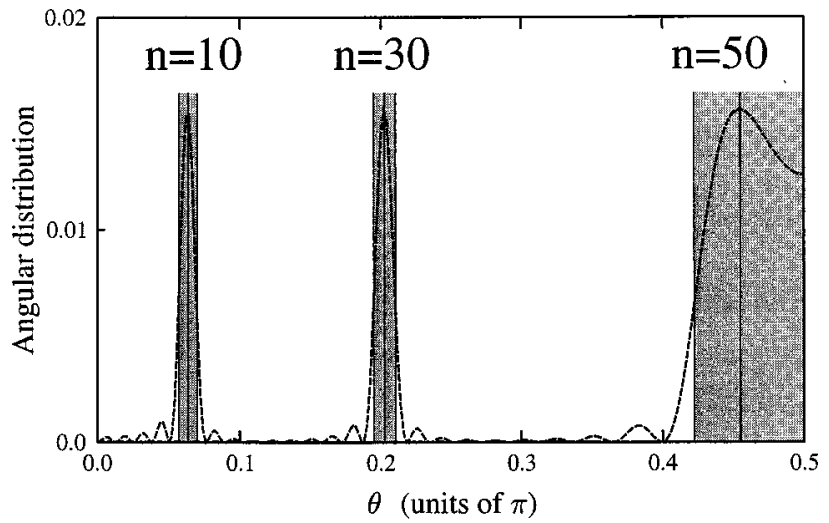

FIG. 1. Comparison between the CTMC method (shaded area) and diffraction according to Ref. 13 (dashed curves) for angular distributions of an electron with $k_{F}=50.5 \pi / w$ and different mode numbers $n$ injected from a quantum wire into a semi-infinite cavity.

where $\bar{F}_{\alpha}$ is the average classical action for each bundle. The amplitude factor for each bundle $H_{\alpha}$ is

$$
\begin{aligned}
H_{\alpha}\left(k_{F}, m, n\right)= & \int d y_{2} \int d y_{1} \phi_{n}\left(y_{2}\right) \phi_{m}\left(y_{1}\right) \\
& \times\left|D_{\alpha}\left(y_{1}, y_{2}, k_{F}\right)\right|^{1 / 2} \times \exp \left[i F_{\alpha}\left(y_{1}, y_{2}, k_{F}, B\right)\right. \\
& \left.-\bar{F}_{\alpha}\left(k_{F}, B\right)\right] .
\end{aligned}
$$

For small mode numbers $m$ and $n$, the integrand in $H_{\alpha}$ of Eq. (2) is, in general, a weakly varying function of $y_{1}$ and $y_{2}$. Depending on the phase space structure of the bundle, the integration over $y_{1}$ and $y_{2}$ may extend over only a fraction of the lead width. The integration amounts to the inclusion of nonclassical paths connecting the entrance and exit leads with the interior of the cavity, which represents diffraction of the wave at the entrance and exit leads. The actions entering Eqs. (1) and (2) are given by

$$
\begin{aligned}
F_{\alpha}\left(y_{1}, y_{2}, k_{F}, B\right)= & \bar{F}_{\alpha}\left(k_{F}, B\right)+k_{F}\left(-\sin \theta_{1} \Delta y_{1}\right. \\
& \left.+\sin \theta_{2} \Delta y_{2}\right),
\end{aligned}
$$

where $\Delta y_{i}=y_{i}-\bar{y}_{i}$ with $\bar{y}_{i}$ the mean value of $y_{i}(i=1,2)$ for the bundle $\alpha$ and

$$
\bar{F}_{\alpha}\left(k_{F}, B\right)=k_{F} L_{\alpha}(B)-B a_{\alpha}(B) / c .
$$

In Eq. (4), $a_{\alpha}$ is the gauge-invariant area enclosed by the open path $\alpha$ and two virtual path segments $\left(\Gamma_{1}\right.$ and $\left.\Gamma_{2}\right)$,

$$
a_{\alpha}(B)=\frac{1}{B} \oint_{\alpha+\Gamma_{2}+\Gamma_{1}} \vec{A} d \vec{q}
$$

The latter two contributions represent the gauge phases of the leads relative to the gauge of the interior of the structure.

The magnetoconductance autocorrelation function is defined as

$$
\begin{aligned}
C(\Delta B) & =\langle\delta T(B+\Delta B) \delta T(B)\rangle_{B} \\
& =\sum_{m, n, m^{\prime}, n^{\prime}}\left\langle\delta T_{m n}(B+\Delta B) \delta T_{m^{\prime} n^{\prime}}(B)\right\rangle_{B},
\end{aligned}
$$

where the bracket stands for the average over $B$ and

$$
\delta T_{m n}(B)=T_{m n}(B)-\left\langle T_{m n}\right\rangle_{B}
$$

where $T_{m n}(B)=\left|t_{m n}(B)\right|^{2}$ is the transmission coefficient. Using the semiclassical expression of $t_{m n}$ [Eqs. (1-3)], we calculate the transmission coefficient from which $\delta T$ is obtained by subtracting the averaged background $\langle T\rangle_{B}$ in Eq. (8) in terms of a third order polynomial fit as in Ref. 2. Thus the present SC calculation of $C(\Delta B)$ includes fluctuations off-diagonal in both mode numbers $\left(m \neq m^{\prime}\right.$ and $\left.n \neq n^{\prime}\right)$ and bundle numbers and therefore avoids the so-called "diagonal" approximation to $C(\Delta B){ }^{8}$

In the limit of large mode numbers, the semiclassical evaluation of $C(\Delta B)$ can be considerably simplified when we employ the PSC approximation taking, however, diffraction effects partially into account through the binning technique of the CTMC method. In the customary PSC approximation, the summation over trajectories in Eq. (1) extends over classical paths whose incoming and outgoing angles, $\theta_{1}$ and $\theta_{2}$, must satisfy the conservation of transverse momenta at the entrance and exit leads,

$$
\sin \theta_{1}=\frac{ \pm m \pi}{k_{F} w}, \quad \sin \theta_{2}=\frac{ \pm n \pi}{k_{F} w},
$$

where $w$ denotes the lead width. The CTMC method maps the quantum numbers of the wires onto the classical angular distribution inside the cavity in terms of bins of a continuous classical phase-space variable in the transverse degree of freedom according to microcanonical ensemble. Each quantum number $m$ corresponds to the uniform distribution of transverse momentum or, equivalently, of $\sin \theta$ within the bin,

$$
\left|k_{F} \sin \theta-\frac{ \pm m \pi}{w}\right| \leqslant \frac{\pi}{w} .
$$

For the few-mode case, the distribution function depends on the choice of the binning in the CTMC method. However, in the high-mode limit, for which the PSC is expected to be valid, the correspondence becomes unique. Figure 1 gives a comparison between the quantum angular distribution for an electron injected from a wire into a semi-infinite half space with $k_{F}=50.5 \pi / \mathrm{w}$ and the corresponding CTMC distribution [Eq. (10)]. Obviously, the CTMC distribution approximates the dominant diffraction peaks quite well.

Within the PSC approximation and employing the diagonal approximation, ${ }^{8}$ the sum over pairs of bundles can be converted to an integral over area differences $a$,

$$
C(\Delta B)=\sum_{m, n} \int_{-\infty}^{\infty} d a P_{2}^{m n}\left(a=a_{\alpha^{\prime}}-\left.a_{\alpha}\right|_{\alpha \neq \alpha^{\prime}}\right) \exp \left(\frac{i}{c} \Delta B a\right),
$$

where $P_{2}^{m n}$ denotes the probability distribution of pairs of bundles entering the cavity with angles belonging to bin $m$ and exiting with angles belonging to bin $n$. In Eq. (11) we have employed a Taylor expansion of $F_{\alpha}\left(y_{1}, y_{2}, k_{F}, B\right)$ [Eq. (3)] in B. It was found that the variations of $F_{\alpha}$ due to the B field dependence of $L_{\alpha}$ and $a_{\alpha}$ cancel within a given bundle, leaving the exponent in Eq. (11) as the leading term to order $\Delta B$. Through $P_{2}^{m n}$, the angular correlation between pairs of 


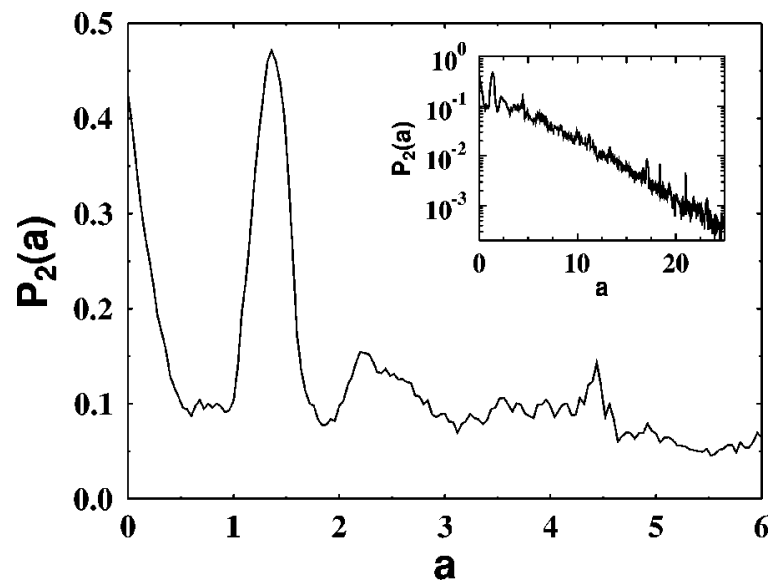

FIG. 2. Area pair distribution for stadium with two $90^{\circ}$ leads (see inset of Fig. 3 with $l / 2=R=1$ and $w=0.4$ ). The peak around $a=1.36$ comes mainly from the area difference between the directpath bundle and a bundle bouncing 3 times within the cavity (a representative trajectory is shown as the inset of Fig. 3). Inset: exponential decay of $P_{2}(a)$ at larger value of $a$.

trajectories is explicitly taken into account. Previous approximations have neglected the correlation between areas and angles assuming ergodic mixing in chaotic cavities. This assumption breaks down for short trajectories even in a strongly chaotic cavity such as the stadium studied here.

We have calculated the area pair distribution $P_{2}^{m n}(a)$ using a large number of trajectories $\left(>10^{7}\right)$ with initial conditions uniformly distributed in transverse phase space. Figure 2 shows the calculated $P_{2}(a)=\sum_{m, n} P_{2}^{m n}(a)$ for a stadium with $90^{\circ}$ leads and lead width $w=0.4$ (see the inset of Fig. 3) summed over 50 angle bins. We have checked for the convergence as a fraction of bin size and number of trajectories. For larger $a, P_{2}(a)$ decays exponentially, as discussed in the previous studies. ${ }^{8-10}$ At small and intermediate values of $a$, however, the distribution function is highly structured and a prominent peak occurs at $a=1.36$ (lengths are measured in units of the radius $R$ of the semicircle of the stadium). By tracking the trajectory information, we can identify bundle pairs which contribute significantly to this peak. It consists primarily of the bundle of direct paths and a bundle with three bounces inside the cavity (see Fig. 3, inset).

The magnetoconductance autocorrelation function, shown in Fig. 3, is calculated by both methods discussed above, i.e., the full semiclassical expression [Eq. (7)] and the simplified expression using PSC with binning technique [Eq. (11)]. The calculation is performed for $k_{F}=3.5 \pi / w$ for the former while the correlation function is obtained by performing Fourier transform of $P_{2}(a)$ in Fig. 2 for the latter method, i.e., for the high-mode limit. Interestingly, both calculations display similar characteristic features: (a) an approximate (squared) Lorentzian slope for small $\Delta B$ which results from the exponential tail in $P_{2}(a)$ (Fig. 2, inset) in agreement with previous findings and (b) damped oscillations for larger $\Delta B$ which were absent in previous semiclassical models. The oscillations are caused by interference between short trajectories. The presence of oscillations appears to be quite general and has been confirmed by applying our semiclassical theories to other geometries with different shapes of the mi-

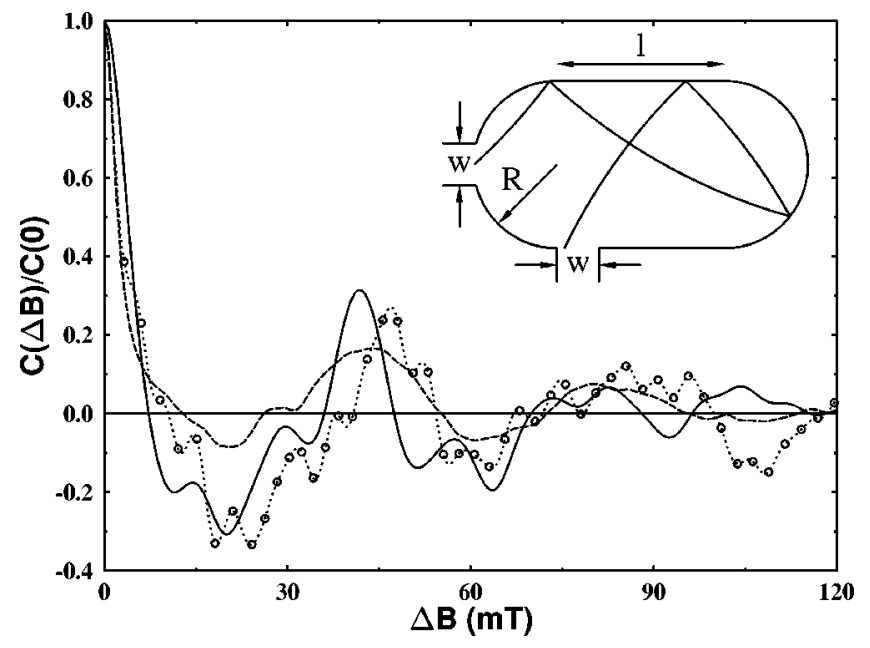

FIG. 3. Magnetoconductance autocorrelation function for the stadium. Dotted curve: experimental data. Solid curve: full semiclassical result; dashed curve: Fourier transform of $P_{2}(a)$ in Fig. 2. Inset: geometry of stadium $(l / 2=R=1$ and $w=0.4)$ and a threebounce trajectory contributing the peak at $a=1.36$ in $\mathrm{P}_{2}(a)$. For unit conversion we used the area of the stadium in the experiment to be $0.5 \mu \mathrm{m}^{2}$.

crostructure and different orientations of the leads. For the present microstructure, there is one dominant component of the oscillation with frequency $\Delta B=41 \mathrm{mT}$. Within the CTMC calculation, this frequency can be directly traced to the strong peak in the pair distribution $P_{2}(a)$ at $a=1.36$. The semiclassical results are also compared with experimental data for three open modes. All observed features agree qualitatively well with the experiment which is described in more detail in Ref. 2. For our comparison, we have determined the autocorrelation function $C(\Delta B)$ from the experimental and theoretical conductance data for the same interval of the magnetic field $(0.01 T \leqslant B \leqslant 0.15 T) .{ }^{15}$ The full evaluation of the SC amplitude, which is expected to be valid at low modes, agrees better with the experiment not only in the qualitative features but also in the amplitude of higher Fourier components. We have also verified that the oscillatory structure is still present after averaging over a range of $k_{F}$ values which models the thermal average in the experiment.

The comparison between the full semiclassical treatment and the simplified PSC calculation indicates that the presence of oscillations is independent of the diagonal approximation. However, the PSC approximation can reproduce the oscillations in the magnetoconductance autocorrelation function only when the pair distribution $P_{2}$ is calculated with the CTMC binning, i.e., only when pairs of angular correlated trajectories are taken into account. Without binning, the pronounced structures in $P_{2}(a)$ average out and $C(\Delta B)$ becomes essentially positive definite. We note that the semiclassical $C(\Delta B)$ obtained from $\delta T$ does not necessarily agree with the one from $\delta R$, where $R$ is the reflection probability. To restore the symmetry between $\delta R$ and $\delta T$, "ghost" orbits are required ${ }^{12}$ which are beyond the present semiclassical approximations.

In summary, we have introduced new semiclassical descriptions of the magnetoconductance autocorrelation function $C(\Delta B)$ through ballistic microstructures. We develop a 
modified semiclassical approximation which employs gaugeinvariant areas, sums explicitly over bundles of paths rather than individual trajectories, eliminates the stationary-phase approximation for the integration over leads by including diffraction effects, and avoids the diagonal approximation. In order to extract a simple physical picture of the classical origin of the structures in $C(\Delta B)$, we introduce a simplified description based on the primitive semiclassical approximation (PSC) combined with the CTMC binning technique for mapping of the classical phase space onto mode numbers in quantum wires. Within these semiclassical approaches, the characteristic oscillations of magnetoconductance autocorrelation functions resulting from short-path trajectories can be reproduced. We find these oscillations to be quite general and determined by one or several peaks in the area pair distribution $P_{2}(a)$ at small $a$. Our results are compared favorably with experiments.

C.M. acknowledges collaboration with Professor R. Westervelt on the experiment. This work was supported by the NSF and U.S. DOE Office of BES, Division of Chemical Science, under Contract No. DE-AC05-96OR22464 with LMERC.
*Present address: Institute for Theoretical Physics, Technical University of Vienna, A-1040 Vienna, Austria.

${ }^{1}$ See, e.g., Quantum Transport in Ultrasmall Devices, edited by D. Ferry, H. Grubin, C. Jacobini, and A.-P. Jauho, Vol. 342 of NATO Advanced Studies Institute Series B: Physics (Plenum, New York, 1995).

${ }^{2}$ C. M. Marcus, R. M. Westervelt, P. F. Hopkins, and A. C. Gossard, Phys. Rev. Lett. 69, 506 (1992).

${ }^{3}$ M. W. Keller, O. Millo, A. Mittal, D. E. Prober, and R. N. Sacks, Surf. Sci. 305, 501 (1994).

${ }^{4}$ C. M. Marcus, A. J. Rimberg, R. M. Westervelt, P. F. Hopkins, and A. C. Gossard, Surf. Sci. 305, 480 (1994).

${ }^{5}$ L. A. Bunimovich, Funct. Anal. Appl. 8, 254 (1974); G. Benettin and J. M. Strelcyn, Phys. Rev. A 17, 773 (1978).

${ }^{6} \mathrm{M}$. Gutzwiller, Chaos in Classical and Quantum Mechanics (Springer Verlag, New York, 1991), and references therein.
${ }^{7}$ R. A. Jalabert, H. U. Baranger, and A. D. Stone, Phys. Rev. Lett. 65, 2442 (1990).

${ }^{8}$ H. U. Baranger, R. A. Jalabert, and A. D. Stone, Chaos 3, 665 (1993).

${ }^{9}$ R. V. Jensen, Chaos 1, 101 (1991).

${ }^{10}$ W. A. Lin, J. B. Delos, and R. V. Jensen, Chaos 3, 655 (1993).

${ }^{11}$ W. A. Lin and R. Jensen, Phys. Rev. B 53, 3638 (1996).

${ }^{12}$ C. Schwieters, J. A. Alford, and J. B. Delos, Phys. Rev. B 54, 10 652 (1996)

${ }^{13}$ L. Wirtz, J. Z. Tang, and J. Burgdörfer, Phys. Rev. B 56, 7589 (1997).

${ }^{14}$ H. Ishio and J. Burgdörfer, Phys. Rev. B 51, 2013 (1995).

${ }^{15}$ Note the interval for $C(\Delta B)$ shown in Fig. 2(a) of Ref. 2 is somewhat larger $(0.01 T \leqslant B \leqslant 0.29 T)$ but the oscillatory structure is similar. 